\title{
The DMTPC detector
}

\section{G. Sciolla*}

Massachusetts Institute of Technology

E-mail: sciolla@mit.edu

J. Battat, T. Caldwell, B. Cornell, D. Dujmic, P. Fisher, S. Henderson, R. Lanza, A. Lee, J. Lopez, A. Kaboth, G. Kohse, J. Monroe, T. Sahin, R. Vanderspek, R. Yamamoto, H. Yegoryan

Massachusetts Institute of Technology

\section{S. Ahlen, D. Avery, K. Otis, A. Roccaro, H. Tomita}

Boston University

\section{A. Dushkin, H. Wellenstein}

Brandeis University

Directional Dark Matter detectors have the potential of yielding an unambiguous observation of WIMPs even in presence of insidious background. In addition, by measuring the direction of the Dark Matter particles such detectors can discriminate between the various models that describe Dark Matter in our galaxy. The DMTPC detector is a novel directional DM detector consisting of a low-pressure $\mathrm{CF}_{4}$ time projection chamber with optical readout. Recent measurements proved that this technology is able to reconstruct the energy, direction, and sense of the lowenergy nuclear recoils produced by neutrons from a ${ }^{252} \mathrm{Cf}$ source, as well as efficiently reject electromagnetic backgrounds. A 10-liter DMTPC detector is ready for underground operation. A $1 \mathrm{~m}^{3}$ detector, now in the design phase, will soon allow us to improve the existing limits of SD-interactions of WIMPs on protons by over one order of magnitude.

Identification of dark matter 2008

August 18-22, 2008

Stockholm, Sweden

\footnotetext{
* Speaker.
} 


\section{Directional Dark Matter detectors}

Directional Dark Matter (DM) detectors [1] are designed to detect the daily modulation in the direction of the Dark Matter wind [2]. The direction of the incoming WIMP is encoded in the direction of the nuclear recoil originated by the elastic scattering of the WIMPs with the atoms in the detector. A precision of 20-30 degrees can be obtained using a very dilute gas (40-100 torr) as a target material. Directional detectors offer unique ways to suppress a variety of backgrounds. The correlation between the energy and length of the nuclear recoil is very powerful in suppressing backgrounds from photons, electrons, and alpha particles. The determination of the direction of the nuclear recoils is used to discriminate between signal (correlated with the direction of the DM wind), neutron backgrounds (randomly distributed around the detector), and solar neutrino backgrounds [3] (pointing back to the Sun).

Finally, the determination of the direction of arrival of Dark Matter particles can discriminate between various DM halo distributions in our Galaxy[4], making DM directional detectors unique observatories for underground WIMP astronomy.

\section{The DMTPC detector}

The DMTPC detector is a low-pressure time projection chamber (TPC) with optical readout. The TPC is filled with tetrafluoromethane $\left(\mathrm{CF}_{4}\right)$ at a pressure of about 50 torr. At such a pressure, a typical collision of a WIMP with a gas molecule would cause a nucleus to recoil by about $2 \mathrm{~mm}$. The ionization electrons produced by the recoiling nucleus drift in the gas along the electric field toward the amplification region. In the amplification process, scintillation photons are produced together with electrons. A CCD camera mounted above the cathode mesh records these photons to produce an image of the nuclear recoil track as projected on the amplification plane. Because the energy loss is not uniform along the trajectory, one can determine the direction of the incoming WIMP ("head-tail" measurement). An array of photomultipliers (PMTs) mounted above the cathode mesh measures the length of the recoil in the drift direction.

$\mathrm{CF}_{4}$ was chosen as target material primarily because of its high content in fluorine. ${ }^{19} \mathrm{~F}$ is an ideal element [5] to detect spin-dependent interactions on protons, due to its large spin factor and isotopic abundance. In addition, $\mathrm{CF}_{4}$ is an excellent detector material $[6,7]$, with good scintillation properties [8] and low transverse diffusion. Finally, $\mathrm{CF}_{4}$ is non-flammable and non-toxic, and, therefore, safe to operate underground.

Electron amplification is obtained by applying a large potential difference $(\Delta \mathrm{V}=0.6-1.1 \mathrm{kV})$ between two conductive woven meshes (or between a mesh and a copper plate) separated by about $500 \mu \mathrm{m}$. The meshes are made of $28 \mu \mathrm{m}$ stainless steel or $\mathrm{Cu}$ wire, with a pitch of $256 \mu \mathrm{m}$, which determines the intrinsic spatial resolution of the detector. The CCDs provide 2-D readout at a very low cost per channel, which makes directional detectors economically viable.

\section{Measurement strategy}

The DMTPC detector simultaneously measures the number of photons collected the CCD camera, the projection of the recoiling nucleus along the amplification plane, the energy loss along 
the recoil track, the width and integral of the PMT signal, the electronic signal produced on the amplification plane.

The energy of the nuclear recoil can be independently reconstructed from the number of photons observed in the CCDs, the integral of the electronic signal produced on the amplification mesh, and the integral of the PMT signal. The track length of the recoiling nucleus is reconstructed by combining the measurement of the projection along the amplification plane (from pattern recognition in the $\mathrm{CCD}$ ) and the projection along the direction of drift, determined from the width of the signal recorded in the PMTs. The sense of the recoil track can be determined by the Bragg curve, the characteristic variation of the energy deposit along the length of the track.

The combination of these measurements reconstructs the energy, direction, and sense of nuclear recoils from WIMPs, allowing for an excellent rejection of the electromagnetic backgrounds. The gamma ray rejection factor, measured using a ${ }^{137} \mathrm{Cs}$ source, is better than 2 parts per million.

The CCD images has a long ( 1 second) exposure. When a trigger is generated by the PMT or the electronic readout of the amplification plane, the CCD is read-out and the event is saved to disk. Otherwise, the CCD is simply reset, to minimize dead time.

\section{Current prototype and $R \& D$ results}

The current DMTPC prototype (Figure 1, top left) consists of two optically independent regions contained in one stainless steel vessel. Each region is a cylinder with $25 \mathrm{~cm}$ diameter and $25 \mathrm{~cm}$ height contained inside a field cage. The amplification is obtained by using a mesh-plate design. The detector is read-out by two CCD cameras, each imaging one drift region. The optical system uses two Nikon photographic lenses with f-number of 1.2 and focal length of $55 \mathrm{~mm}$, and two Apogee U6 cameras equipped with Kodak 1001E CCD chips. Because the total area imaged is $16 \times 16 \mathrm{~cm}^{2}$, the detector has an active volume of about 10 liters.

An ${ }^{241} \mathrm{Am}$ source producing $5.5 \mathrm{MeV}$ alpha particles is used to study the gain of the detector as a function of the voltage in the amplification region and gas pressure. Typical gas gains are $\approx$ $10^{5}$. We also measure the transverse diffusion as a function of the primary electrons drift distance These studies show that the transverse diffusion is less then $1 \mathrm{~mm}$ for a drift distance of $25 \mathrm{~cm}$ [9].

The ability of the DMTPC detector to determine the sense and direction of nuclear recoils has been evaluated by studying the recoil of fluorine nuclei in interaction with low-energy neutrons. For nuclear recoils with energy below $1 \mathrm{MeV}$, the energy deposition decreases along the path of the recoil, allowing for the identification of the "head" ("tail") of the event by a smaller (larger) energy deposition. The initial measurements used $14 \mathrm{MeV}$ neutrons from a deuteron-triton generator. The reconstructed recoils with energy between 200 and $800 \mathrm{keV}$ allowed us to observe the "head-tail" effect with a significance of $8 \sigma$ [9].

In subsequent measurements [10] we used lower energy neutrons generated by a ${ }^{252} \mathrm{Cf}$ source. To achieve a 2-D reconstruction of the nuclear recoils, a mesh-based detector was utilized. Better sensitivity to lower energy thresholds was achieved by lowering the $\mathrm{CF}_{4}$ pressure to 75 torr. Figure 1 (bottom left) shows a Cf-induced nuclear recoil reconstructed in the DMTPC detector. The neutron was traveling right to left. The decreasing $d E / d x$ along the track direction, clearly visible in the image, proves that the detector is sensitive to the sense of the direction on an event-by-event basis. Measurements of the length and asymmetry of the recoil tracks as a function of their energy 



Figure 1: Top left: image of the 10-liter DMTPC prototype. Bottom left: image of a nuclear recoil from a low-energy neutron in $\mathrm{CF}_{4}$ at 75 torr. Right: expected sensitivity (90\% C.L.) to spin-dependent WIMP scattering on protons for a DMTPC detector (solid lines) compared with published limits from other experiments (dashed lines). The shaded area shows the MSSM favored region.

show good agreement with the predictions of the simulation and prove good head-tail discrimination for recoils above $100 \mathrm{keV}$ [10]. The "head-tail" discrimination is expected to extend to recoils above $50 \mathrm{keV}$ when the detector is operated at a pressure of 50 torr.

\section{Future detectors and expected sensitivity}

A $1-\mathrm{m}^{3}$ DMTPC detector is being designed. The apparatus consists of a stainless steel vessel of $1.3 \mathrm{~m}$ diameter and $1.2 \mathrm{~m}$ height. Nine CCD cameras and nine PMTs are mounted on each of the top and bottom plates of the vessel, separated from the active volume of the detector by an acrylic window. The detector consists of two optically separated regions. Each of these regions is equipped with a triple-mesh amplification device, mounted in between two symmetric drift regions. Each drift region has a diameter of $1.2 \mathrm{~m}$ and a height of $25 \mathrm{~cm}$, for a total active volume of $1 \mathrm{~m}^{3}$. A field cage made of stainless steel rings keeps the uniformity of the electric field within $1 \%$ in the fiducial volume. A gas system recirculates and purifies the $\mathrm{CF}_{4}$. 
When operating the detector at a pressure of 50 torr at 21 degrees $\mathrm{C}$, this module will contain $250 \mathrm{~g}$ of $\mathrm{CF}_{4}$. Assuming a data-taking efficiency of $50 \%$, a one-year run will yield $45 \mathrm{~kg}$-days.

The sensitivity of the DMTPC detector to SD interactions of WIMPs on protons has been studied for exposures of 0.1 and $100 \mathrm{~kg}$-years (Figure 1, right). We assumed that the detector will be operated at a depth of 2,000 m.w.e. inside a $40 \mathrm{~cm}$ thick polyethylene neutron shielding, and the absence of internal backgrounds above the $50 \mathrm{keV}$ threshold. This study shows that improvements of a factor 50 over the existing measurements can be obtained by operating a $1-\mathrm{m}^{3}$ DMTPC detector for less than a year. A larger detector, with an active mass of $10^{2}-10^{3} \mathrm{~kg}$, will be able to explore a significant portion of the MSSM parameter space. This detector is an ideal candidate for the DUSEL laboratory in South Dakota.

\section{Conclusion}

The measurement of the direction of the incoming WIMPs by a directional Dark Matter detector can provide an unambiguous positive observation of Dark Matter particles as well as a unique tool for underground WIMP astronomy.

Our DMTPC detector is designed to measure the energy, direction, and sense of the nuclear recoils produced in elastic collisions of WIMPs in low-pressure $\mathrm{CF}_{4}$ gas. The combination of these measurements allows for excellent background rejection, while the use of an optical readout substantially reduces the costs, making a ton-size directional detector economically viable. The choice of $\mathrm{CF}_{4}$ as the target material makes this detector well suited for studies of spin-dependent interactions.

\section{Acknowledgments}

The DMTPC project is supported by the DoE ADR program, the National Science Foundation, the Pappalardo Fellowship program, and the MIT Kavli Institute and Physics Department.

\section{References}

[1] G. Sciolla, submitted to Modern Physics Letters A, arXiv:0811.2764 [astro-ph].

[2] D. N. Spergel, Phys. Rev. D 37, 1353 (1988).

[3] J. Monroe and P. Fisher, Phys. Rev. D 76, 033007 (2007).

[4] M. S. Alenazi and P. Gondolo, Phys. Rev. D 77, 043532 (2008).

[5] R. J. Ellis and R. A. Flores, Phys. Lett. B 263, 259 (1991).

[6] A. Pansky et al., Nucl. Instrum. Meth. A 354, 262 (1995).

[7] L. G. Christophorou, et al., J. Phys. Chem. Ref. Data 25, 1341 (1996)

[8] A. Kaboth et al., [DMTPC Collaboration], Nucl. Instrum. Meth. A 592, 63 (2008).

[9] D. Dujmic et al., [DMTPC Collaboration], Nucl. Instrum. Meth. A 584, 327 (2008).

[10] D. Dujmic et al., [DMTPC Collaboration], Astropart. Phys. 30, 58 (2008). 